\title{
Travel time and cost analysis of PAVE application in Malaysia
}

\begin{abstract}
Transportation has already become a huge necessity in people's life today. As the world progresses, transportation systems will have to undergo paradigm shifts to adapt with the changing requirements. Personal transportation is typically preferred to the public transportation and to date, most domestic travels have been made on the ground through roads. However, with the increasing traffic volume, the average time spent travelling on roads has significantly increased for the same amount of distance travelled. It has been proposed that utilization of the possible third dimension of transportation system, which is the air transportation, can aid personal transportation and alleviate this problem. This notion gives birth to the design concepts of the personal air vehicle (PAVE) that is envisioned to operate synergistically with the existing ground and air infrastructures. This paper aims to preliminarily study and analyze the potential benefits of having PAVE option in Malaysia for domestic travel and how its performance fares in comparison to existing transportation options. The results of travel time and cost comparisons highlight the potential of PAVE application for the domestic transportation in Malaysia, particularly for personal travel need.
\end{abstract}

Keyword: Dual-mode car; Flying car; PAVE; Personal air vehicle; Personal travel 